\title{
Continuous Intra-intestinal Infusion of Levodopa/Carbidopa in Advanced Parkinson's Disease
}

\author{
a report by \\ Dag Nyholm ${ }^{1}$ and Per Odin ${ }^{2}$ \\ 1. Department of Neuroscience, Neurology, Uppsala University Hospital, Uppsala, Sweden; \\ 2. Department of Neurology, Central Hospital, Bremerhaven, Germany
}

DOI:10.17925/ENR.2007.00.01.45

The concept of continuous dopaminergic stimulation (CDS) is presently the aim of most pharmacotherapy for Parkinson's disease (PD). The theoretical background to CDS is that the physiological striatal dopaminergic stimulation is constant and that oral therapy with levodopa in PD can replace dopaminergic stimulation only in an intermittent, non-physiological manner. ${ }^{1,2}$ This pulsatile stimulation of the dopamine receptors is thought to contribute to the development of motor fluctuations, which are common after a few years of levodopa treatment and may substantially affect quality of life. ${ }^{3,4}$ Oral levodopa is highly effective but cannot be delivered in a controlled way because of its short plasma half-life and its erratic absorption due to slowed gastric emptying. ${ }^{5}$ Dopamine agonists for oral administration have longer plasma half-lives than levodopa, thereby causing less pulsatile stimulation. However, the efficacy of the agonists is only partial compared with levodopa, and side effects are more common. Levodopa is therefore almost always needed in advanced PD.

In patients in whom all conventional pharmacotherapy has been tried, three main invasive therapies remain: subcutaneous infusion of apomorphine; intra-intestinal infusion of levodopa; and deep-brain stimulation (DBS). The choice of therapy involves striking a balance between the best possible symptom relief and a minimum of side effects.

This report will describe the use of intra-intestinal infusion of levodopa. The first study of duodenal infusion of levodopa was published in $1986^{6}$ and confirmed the stabilising effects on motor fluctuations that had previously been demonstrated in studies with intravenous infusion.?

\section{The Drug Delivery System}

The first studies of intravenous and intraduodenal infusions of levodopa utilised water solutions of levodopa. The chemical properties of

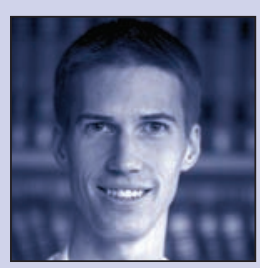

Dag Nyholm is a Resident in Neurology in the Department of Neuroscience and Neurology at Uppsala University Hospital, Sweden. He has recently received a grant from the Swedish Society for Medical Research, intended for two years' parttime research in the fields of Parkinson's disease and amyotrophic lateral sclerosis.

E: dag.nyholm@neurologi.uu.se

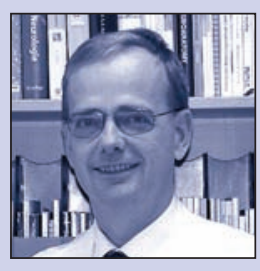

Per Odin is Chairman of the Department of Neurology at Central Hospital, Bremerhaven, Germany. His focus is on movement disorders, especially Parkinson's disease and restless legs. levodopa and carbidopa require large volumes of water for preparing one day's supply, which made the method laborious. ${ }^{7}$ This problem was addressed with the development of a levodopa/carbidopa gel (Duodopa ${ }^{\circledR}$, Solvay Pharmaceuticals $\mathrm{GmbH}$, Hannover, Germany). By using a gel of carboxymethylcellulose (2.92\% carmellose sodium), the concentration of levodopa is $20 \mathrm{mg} / \mathrm{ml}$, making a cassette containing $100 \mathrm{ml}$ enough for one day's use for most patients. The cassette is attached to a portable pump (CADD-Legacy-Duodopa, Smiths Medical, MN, US). The tube of the cassette is connected to a percutaneous endoscopic gastrostomy (PEG) tube, containing a smaller-bore intestinal tube inside (Bengmark tube, Nutricia, Switzerland). The intestinal tube is placed beyond the pylorus to allow for immediate absorption across the intestinal mucosa in the duodenum or proximal jejunum. A nasoduodenal tube may be used during a test period to avoid unnecessary surgery.

\section{Dosage}

Initial dosage of the levodopa/carbidopa gel is calculated based on the previous dose of oral levodopa or levodopa equivalents. The dose is

Oral levodopa is highly effective but cannot be delivered in a controlled way because of its short plasma half-life and its erratic absorption due to slowed gastric emptying.

usually between 20 and $120 \mathrm{mg} / \mathrm{h}$ depending on the individual patient's requirements. An individual morning bolus dose, usually 40-200mg levodopa, is always used to rapidly reach steady-state, from which the concentration can be kept constant by the individualised infusion rate. Both morning dose and infusion rate are fine-tuned over the course of the first few days to find the optimal dose that produces a continuous 'on' state without troublesome dyskinaesia. Adjustments of the infusion rate can be made in small increments of $2 \mathrm{mg} / \mathrm{h}$. The patient is discharged from the hospital after PEG surgery is completed, an optimal dose is found and the patient - or a relative - has learned how to handle the infusion system. This usually takes about one week. Follow-up should be carried out by a PD-specialised nurse or at an outpatient visit a few weeks later. Dosage may need to be adjusted after weeks to months, probably due to long-term plastic changes in the brain. The levodopa/carbidopa infusion has mostly been used as monotherapy, which is practical, but it may be combined with other anti-Parkinsonian drugs, especially for 'nondopaminergic' symptoms. 


\section{Pharmacokinetics}

Metabolism of levodopa/carbidopa is well known from studies on oral formulations. Levodopa is absorbed from the proximal small intestine (duodenum/jejunum) but not from the stomach; this is why the tip of the intestinal tube must be placed beyond the pylorus. Even if levodopa were continuously infused into the stomach, plasma concentrations would fluctuate significantly more than after duodenal/jejunal infusion.8,9 Fluctuation in plasma concentrations can be described with the coefficient of variation (CV), which is the standard deviation divided by mean plasma concentration. The CV of orally administered levodopa usually ranges from 25 to $70 \%$, even in

\author{
Published studies with levodopa \\ infusions show a decrease in 'off' time \\ and a reduced severity of the \\ symptomatology of the remaining \\ 'off' periods.
}

the presence of catechol-O-methyltransferase inhibitors. ${ }^{9-11} \mathrm{CV}$ of levodopa infused intra-intestinally is in the range of $6-20 \%$ significantly lower than oral formulations.9,12 Mean plasma concentrations producing optimal response vary largely between patients, ranging from 0.5 to $7 \mu \mathrm{g} / \mathrm{ml}$ (i.e. $2.5-35.5 \mathrm{nmol} / \mathrm{ml}$ ).

\section{Effect on Motor and Non-motor Symptoms}

Published studies with levodopa infusions show a decrease in 'off' time and a reduced severity of the symptomatology of the remaining 'off' periods. $^{7}$ For example, Nyholm et al. ${ }^{13}$ published the results of a randomised crossover study comparing treatment with intraduodenal infusion of levodopa/carbidopa alone versus individualised conventional therapy in 24 patients (three weeks on each treatment). On the basis of blinded evaluations of video recordings and electronic diaries, there was an increase in 'on' assessments from 81 to $100 \%$ (median values), a corresponding reduction in 'off' assessments and an improvement of the Unified Parkinson Disease Rating Scale (UPDRS) score from 53 to 35 . The health-related quality of life also improved significantly, as measured by the Parkinson's Disease Questionnaire-39 (PDQ-39) and the 15D Quality of Life instrument. The side effects were comparable in both groups.

In our experience, there is often also a substantial improvement regarding dyskinaesias, in terms of both time with dyskinaesias and dyskinaesia intensity, which develops more slowly during the first months of treatment (unpublished data). The fact that dyskinaesias improve even when similar total levodopa equivalence doses are used confirms that the most important factor behind dyskinaesia is not levodopa itself, but the pulsatile effect resulting from peroral delivery.

Some motor fluctuations may continue to occur, for example in relation to 'off' periods after highly protein-rich meals as well as 'off' periods or dyskinaesia periods associated with stress. It has also been repeatedly observed that patients tend to have rather more 'off' symptoms in the late afternoon/early evening, which is why the administration of a slightly higher drug dose in the afternoon/evening can be considered.

We have also observed improvements regarding psychiatric and psychological aspects of the disease (unpublished observations). Anxiety problems often improve under pump therapy. There also seems to be less tendency for dopaminergic-psychotic side effects under continuous levodopa/carbidopa delivery versus peroral therapy, in spite of similar total levodopa-equivalence doses. This could reflect an advantage to monotherapy levodopa/carbidopa over multiple dopaminergic drugs or continuous stimulation over pulsatile stimulation.

\section{Technical Problems}

The most frequent problems with Duodopa relate to the technical side of the therapy. A common problem is dislocation of the small intestine catheter. Due to displacement of the catheter back into the stomach, fluctuating effects of medication reappear. The catheter position then has to be corrected under radiographic or gastroscopic control.

The catheter may also become blocked or kinked. Blocking can usually be eliminated by flushing the catheter with tap water, but kinks may need to be eliminated by repositioning the catheter.

In rare cases, the PEG or the catheter has become loose or broken in the stomach or the small intestine. If the catheter becomes loose, it normally comes out by itself without further problems and can simply be renewed. A broken PEG puts the patient at risk of complications, such as perforation of the stomach or intestine, and may necessitate open surgery.

The stoma usually heals without significant complications. However, there may be abdominal pain, infection and discharge of gastric juice shortly after the operation. In rare cases, bacterial peritonitis has occurred in connection with the PEG application. The most common chronic local complications are secretion and the formation of hypertrophic granulation tissue. Local infections around the stoma are treated with disinfectants; antibiotic therapy is rarely necessary.

\section{Pharmacological Side Effects}

The side effects of Duodopa are the same as those of treatment with levodopa/carbidopa tablets. Mild nausea and loss of appetite occur The most common chronic local complications are secretion and the formation of hypertrophic granulation tissue.

of dyskinaesia and underactivity in the form of Parkinsonism occur more rarely than with tablet therapy.

ome patients report long-term sedation with levodopa and carbidopa. In addition, sudden sleep episodes (sudden onset of sleep without prior 


\section{Promoting a}

\section{constructive}

dialogue between

science \& society

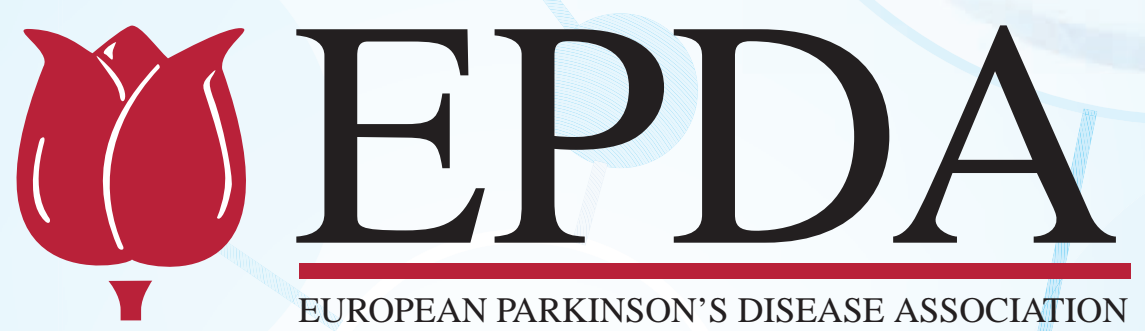

www.epda.eu.com

Supporting the Global Declaration on Parkinson's disease

EPDAPlus

EPNN

JOURNAL

Med Info

Guide to living

with Parkinson's disease
MULTIDISCIPLINARY CONFERENCES

WORLD

PARKINSON'S DISEASE 
tiredness or warning signals) can occur in relation to the treatment, as is the case with other Parkinson's treatments. Patients treated with Duodopa should therefore be advised to take care when driving or using machinery.

Long-term safety follow-up studies show no further unexpected side effects in long-term therapy (up to over 10 years).

\section{Development of Tolerance}

There is no evidence of tolerance developing with Duodopa therapy during the day. On the contrary, the dose can be reduced after the start of therapy in many patients (during the first few weeks or months). The situation is less clear with 24-hour therapy. In this instance, there are sporadic patient reports of possible development of tolerance. ${ }^{7}$ However, most patients under 24-hour therapy do not show any signs of tolerance. ${ }^{14}$

\section{Indications and Contraindications}

The treatment is indicated for PD patients with fluctuations in the effect of oral antiparkinson medication. Peak-dose dyskinaesias can also be improved, and patients will have a maximal effect of L-dopa. There is no age limit and even elderly people can experience beneficial effects. Dementia, however, makes the treatment more difficult to perform and negatively affects the outcome, and should therefore be seen as a relative contraindication. According to the manufacturer, Duodopa should be avoided in patients with hypersensitivity to levodopa or carbidopa,

\section{There is no evidence of tolerance} developing with Duodopa therapy during the day. On the contrary, the dose can be reduced after the start of therapy in many patients (during the first few weeks or months).

glaucoma, serious liver and kidney disease, severe heart failure, severe cardiac arrhythmias and recent stroke.

\section{Duodopa versus Apomorphine Infusion}

There are no comparative studies between Duodopa therapy and continuous subcutaneous infusion of apomorphine with portable pumps. These are both very potent therapies and the clinical experiences indicate that the maximal effects of the two therapies regarding motor symptoms are of a similar degree. It seems that more patients can be treated with monotherapy Duodopa compared with

With levodopa infusion, a surgical
intervention (percutaneous
endoscopic gastrostomy) is necessary;
with apomorphine, this is not the case.

monotherapy apomorphine. This could indicate that the effect of levodopa infusion is slightly stronger or more complete. The most important factors in deciding between these two treatments are, however, the technical-practical aspects and the side effects. With levodopa infusion, a surgical intervention (PEG) is necessary; with apomorphine, this is not the case. With apomorphine infusion, local skin irritation (nodule formation) is frequent; with Duodopa, this is not the case. In patients on apomorphine therapy who develop numerous skin reactions, a change to Duodopa therapy can be considered.

\section{Duodopa versus Deep Brain Stimulation}

There are no comparative studies between Duodopa therapy and DBS available. The clinical experience is that the degree of effect regarding motor symptoms seems comparable. The fact that levodopa infusion has mainly mild and reversible side effects, while DBS has potentially severe and non-reversible side effects, suggests that infusion should at least be considered before DBS is performed. Furthermore, levodopa infusion is an alternative for patients who have absolute or relative contraindications for DBS. This includes elderly patients and patients who are vulnerable from a psychiatric point of view, such as patients with depression.

\section{Summary}

Several research teams have independently demonstrated the advantages of continuous intra-intestinal infusion therapy with the levodopa/ carbidopa gel with regard to constant plasma concentrations and a reduction of motor as well as non-motor symptoms in patients with advanced PD. Levodopa infusion offers a practical solution for patients in whom such a treatment is indicated.
1. Chase TN, Striatal plasticity and extrapyramidal motor dysfunction, Parkinsonism Relat Disord, 2004;10:305-13.

2. Nutt JG, Continuous dopaminergic stimulation: Is it the answer to the motor complications of Levodopa?, Mov Disord, 2007:22:1-9.

3. Ahlskog JE, Muenter MD, Frequency of levodopa-related dyskinesias and motor fluctuations as estimated from the cumulative literature, Mov Disord, 2001;16:448-58.

4. Chapuis S, Ouchchane L, Metz O, et al., Impact of the motor complications of Parkinson's disease on the quality of life, Mov Disord, 2005;20:224-30.

5. Muller T, Erdmann C, Bremen D, et al., Impact of gastric emptying on levodopa pharmacokinetics in Parkinson disease patients, Clin Neuropharmacol, 2006;29:61-7.

6. Kurlan R, Rubin AJ, Miller C, et al., Duodenal delivery of levodopa for on-off fluctuations in parkinsonism: preliminary observations, Ann Neurol, 1986;20:262-5.

7. Nyholm D, Aquilonius SM, Levodopa infusion therapy in Parkinson's disease: state of the art in 2004, Clin Neuropharmacol, 2004;27:245-56.

8. Kurlan R, Nutt JG, Woodward WR, et al., Duodenal and gastric delivery of levodopa in parkinsonism, Ann Neurol, 1988;23: 589-95.

9. Nyholm D, Askmark H, Gomes-Trolin C, et al., Optimizing levodopa pharmacokinetics: intestinal infusion versus ora sustained-release tablets, Clin Neuropharmacol, 2003;26: 156-63.

10. Nutt JG, Woodward WR, Beckner RM, et al., Effect of peripheral catechol-0-methyltransferase inhibition on the pharmacokinetics and pharmacodynamics of levodopa in parkinsonian patients, Neurology, 1994;44:913-19.

11. Nyholm D, Lennernas $H$, Gomes-Trolin C, Aquilonius SM,
Levodopa pharmacokinetics and motor performance during activities of daily living in patients with Parkinson's disease on individual drug combinations, Clin Neuropharmacol, 2002;25: 89-96.

12. Bredberg $E$, Nilsson $D$, Johansson $K$, et al., Intraduodenal infusion of a water-based levodopa dispersion for optimisation of the therapeutic effect in severe Parkinson's disease, Eur J Clin Pharmacol, 1993;45:117-22.

13. Nyholm D, Nilsson Remahl AIM, Dizdar N, et al., Duodenal levodopa infusion monotherapy versus oral polypharmacy in advanced Parkinson disease, Neurology, 2005;64:216-23.

14. Nyholm D, Jansson R, Willows $T$, Remahl IN, Long-term 24-hour duodenal infusion of levodopa: outcome and dose requirements, Neurology, 2005;65:1506-7. 